\title{
Chamas for Change: A Community Based Strategy for Pregnancy, Infancy, and Women Empowerment
}

\author{
Ryan Smith, Laura Ruhl \\ Indiana University School of Medicine Indianapolis, AMPATH, Saving Lives at \\ Birth, Moi Teaching and Referral Hospital
}

Background: Maternal mortality is the leading cause of death among women of childbearing age in Kenya, and 1 in every 19 infants dies before their first birthday. To address this, Chama cha MamaToto was developed as a community-based program for pregnant and breast-feeding women. It integrates health and social education to decrease poor health outcomes and parental stress. It also uses a table banking system called GISHE to develop financial independence. Central to Chamas is peer support and accountability, leading to empowerment of the women. This poster is an analysis of the program's history, and a summary of data collected by the $\mathrm{MNCH}$ team from MTRH.

Results: Women in Chamas had 5 times the odds of delivering in a health facility (OR $=5.07,95 \% \mathrm{Cl}: 2.74-9.39)$. Children in Chamas had $33 \%$ relative reduction in stunting. Women and children in Chamas experienced $30 \%$ absolute reduction in harsh punishment $(p<0.001)$ compared to the control group. The Chamas program showed the potential for combined relative reduction of stillbirths and infant deaths by 56 percent $(p<0.083)$. Additionally, women in Chamas had an $35 \%$ absolute increase in EBF for 6 months.

Personal role: My role working with the Chamas program this summer involved a cost analysis of the program as well as developing an implementation manual.

Conclusion: The next steps for the program include a continued scale up in two new sub counties, validation of findings from a cluster RCT, and a scale up of the program to all of Kenya in 2019. 